\title{
Covariance generation and uncertainty propagation for thermal and fast neutron induced fission yields
}

\author{
Nicholas Terranova ${ }^{1, a}$, Olivier Serot ${ }^{2}$, Pascal Archier $^{2}$, Cyrille De Saint Jean $^{2}$, and Marco Sumini ${ }^{1}$ \\ 1 Industrial Engineering Department, University of Bologna, Italy \\ 2 CEA-Cadarache, DEN, DER, SPRC, 13108 Saint-Paul-lez-Durance, France
}

\begin{abstract}
Fission product yields (FY) are fundamental nuclear data for several applications, including decay heat, shielding, dosimetry, burn-up calculations. To be safe and sustainable, modern and future nuclear systems require accurate knowledge on reactor parameters, with reduced margins of uncertainty. Present nuclear data libraries for FY do not provide consistent and complete uncertainty information which are limited, in many cases, to only variances. In the present work we propose a methodology to evaluate covariance matrices for thermal and fast neutron induced fission yields. The semi-empirical models adopted to evaluate the JEFF-3.1.1 FY library have been used in the Generalized Least Square Method available in CONRAD (COde for Nuclear Reaction Analysis and Data assimilation) to generate covariance matrices for several fissioning systems such as the thermal fission of U235, Pu239 and Pu241 and the fast fission of U238, Pu239 and Pu240. The impact of such covariances on nuclear applications has been estimated using deterministic and Monte Carlo uncertainty propagation techniques. We studied the effects on decay heat and reactivity loss uncertainty estimation for simplified test case geometries, such as PWR and SFR pin-cells. The impact on existing nuclear reactors, such as the Jules Horowitz Reactor under construction at CEA-Cadarache, has also been considered.
\end{abstract}

\section{Introduction}

To obtain maximum benefit from recent simulation tools and neutron transport codes, significant efforts have been spent to improve the accuracy of input data, whose reliability is a fundamental aspect in estimating precise responses for nuclear technology applications [1]. Uncertainties and biases on nuclear reactor integral parameters have to be known with a certain degree of confidence in order to satisfy the safety-by-design standards required in modern nuclear technology. In the present work we are mainly focused on fission yields. Many covariance generation strategies have been developed by several organizations to produce complete uncertainty information for FY [2-5]. However, no covariance values and data formats are available in current nuclear data libraries. In the present work we extensively applied the methodology developed at CEA-Cadarache [6] which aims to faithfully reproduce the existing JEFF3.1.1 library and add consistent covariance information. The results obtained for several fast and thermal neutroninduced fissioning systems have been tested in uncertainty propagation exercises for simple reactor test cases and for the Jules Horowitz Reactor, a high flux material testing facility under construction in the Cadarache site of CEA (France).

\section{Covariance generation}

The covariance generation methodology effectiveness was already demonstrated in [6,7] and it is summarized in Fig. 1. FY covariance matrices have been evaluated using

a e-mail: nicholas.terranova@unibo.it

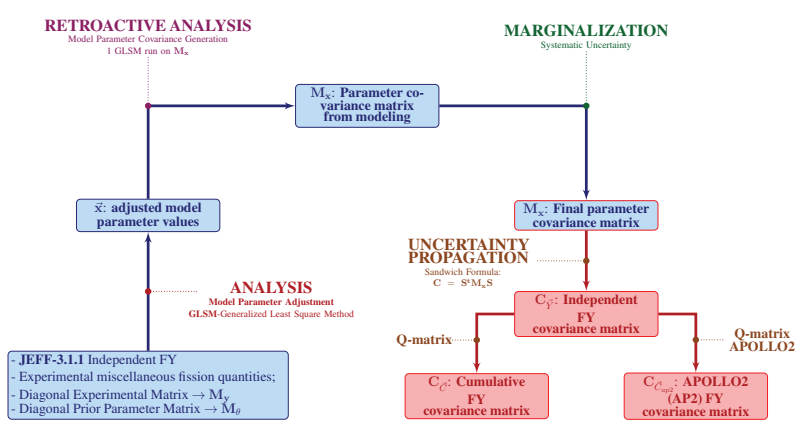

Figure 1. Flow chart of the statistical procedure used to generate covariance matrices for independent and cumulative fission yields.

the CONRAD code [8]. The same independent FY semiempirical models used in JEFF-3.1.1 evaluations to fill the gaps existing in the available experimental measurements were employed and implemented in CONRAD. A Bayesian Generalized Least Square Method (GLSM) has been adopted to find the model parameter best estimates which can faithfully represent the JEFF library.

JEFF-3.1.1 FY values were taken as reference and included in a GLSM procedure as pseudo-experimental data. Actual experimental measurements on miscellaneous fission quantities, such as the total prompt neutron emission probability $P(v)$ and the total prompt neutron multiplicity $\langle v\rangle$, were embedded in the calculation. Preneutron yields were represented using the Brosa fission modes [9]. A simplified prompt neutron emission probability model was employed to obtain post-neutron yields (see Ref. [6]). The Wahl model and the Madland-England

(C) The Authors, published by EDP Sciences. This is an Open Access article distributed under the terms of the Creative Commons Attribution License 4.0 (http://creativecommons.org/licenses/by/4.0/). 


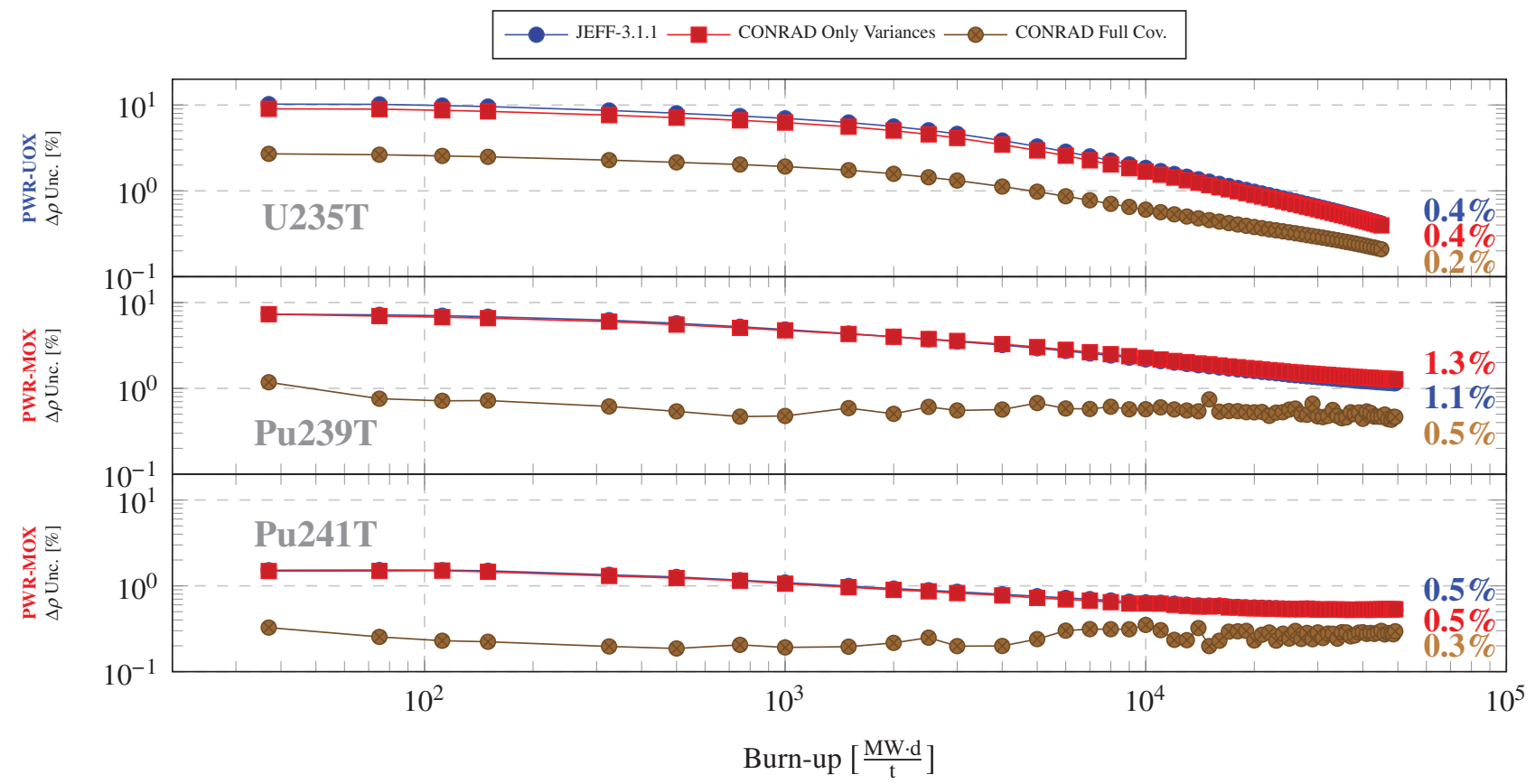

Figure 2. Reactivity loss uncertatinty estimation due to thermal neutron-induced FY data, for UOX and MOX-fueled PWR pin-cells. Results obtained using CONRAD variances are compared to those deriving from JEFF-3.1.1. Using full covariance matrices induces significant uncertainty reductions.

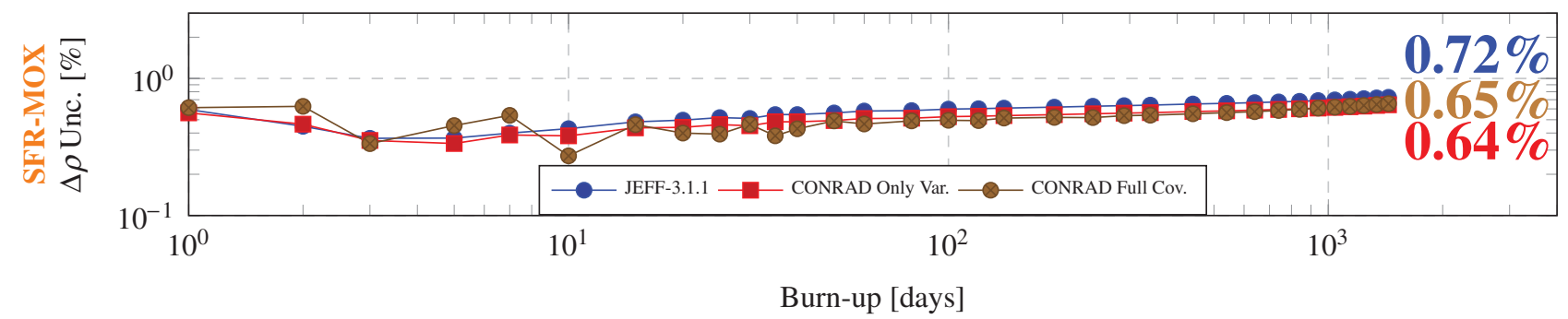

Figure 3. SFR-MOX cell reactivity loss uncertainty estimation due to the fast neutron-induced fission yields of ${ }^{239} \mathrm{Pu}$. Results deriving from CONRAD are compared to those obtained using JEFF-3.1.1 values.

ratio were adopted to calculate respectively isotopic and isomeric FY [10,11]. To properly include systematic uncertainties and correlations the analytical Marginalization technique was used [12]. Such a method allowed us to pursue a nice representation of both the mean values and the uncertainties of the most significant FY contained in JEFF. JEFF-3.1.1 FY were consistently reproduced together with other independent experimental fission quantities. Several fast $(F)$ and thermal $(T)$ neutroninduced FY covariance matrices (U235T, Pu239T, Pu241T, U238F, Pu239F and Pu240F) were generated through this method and included in COMAC (COvariance MAtrices from Cadarache) [13]. In the following section some results on FY uncertainty propagation on integral reactor parameters will be presented.

\section{FY uncertainty propagation}

Our main goal was to test the effects induced by propagating the covariance matrices generated using present methodology in nuclear reactor applications. We used simple direct perturbations to estimate sensitivity coefficients. Since we were concerned on possible nonlinear effects due to the coupling of the Boltzmann and Bateman equations, we compared our results to Monte
Carlo uncertainty propagation estimations (see Ref. [14]) which showed a satisfactory agreement with what was obtained using simple perturbation-derived sensitivities.

Simple test cases were firstly investigated before treating real reactor whole geometries. PWR UOX and MOX pin-cells were considered to test thermal neutroninduced FY uncertainties effects on reactivity and decay heat. SFR-MOX lattice calculations were performed to propagate fast neutron fissioning system yields using the APOLLO3 ${ }^{\circledR}$ code [15]. Uncertainty results showed a satisfactory agreement between JEFF and CONRAD variances, proving a quite global consistency between the two evaluations. Including full covariance matrices allows significant uncertainty reduction essentially due to anti-correlations existing between isobar fission product yields (see for instance Fig. 2). The only exception turned out to be the SFR cell reactivity loss uncertainty, whose value did not seem to be relevantly affected by Pu239F FY uncertainties (see Fig. 3). The JHR, with its high enrichment and flux, presents a reactivity loss uncertainty which is quite affected by U235T FY. CONRAD covariances yielded important uncertainty reduction on reactivity loss, playing certainly a valuable role in fuel cycle length optimization (see Fig. 4). 


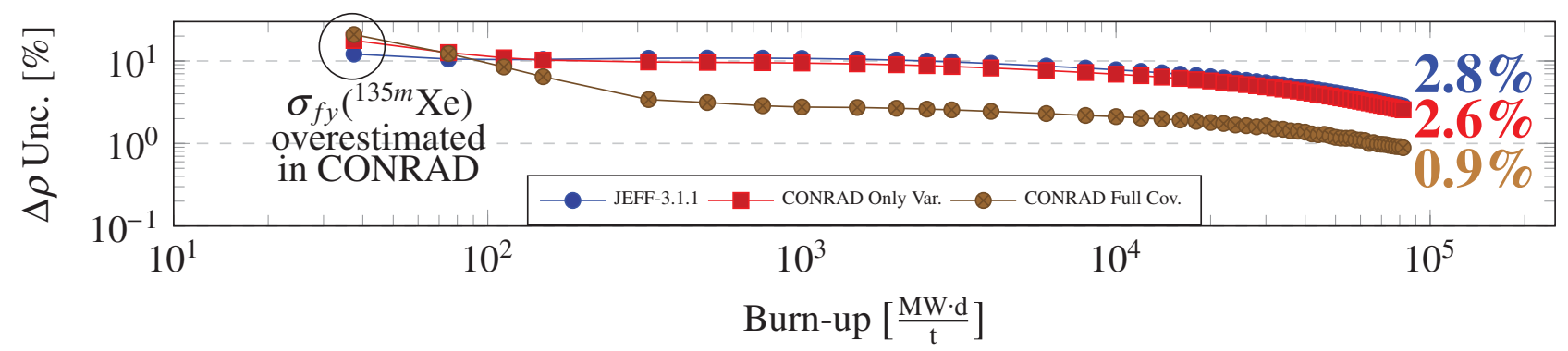

Figure 4. Relative reactivity loss uncertainty as a function of burn-up for the JHR. The propagation of JEFF-3.1.1 ${ }^{235} \mathrm{U}\left(\mathrm{n}_{t h}, \mathrm{f}\right) \mathrm{FY}$ uncertainties has been compared to what we obtain from CONRAD, with and without including correlations.

\section{Conclusions}

FY covariances have been generated using the CONRAD code and trying to reproduce consistently the existing evaluated library JEFF-3.1.1. Covariance results were extensively tested in nuclear reactor applications, showing a satisfactory coherence between CONRAD and JEFF results, and a significant uncertainty reduction induced by CONRAD anti-correlations.

\section{References}

[1] G. Palmiotti et al., Nuclear Data Sheets 118, 596 (2014)

[2] T. Kawano and M.B. Chadwick, Journal of Nuclear Science and Technology 50, 1034 (2013)

[3] L. Fiorito et al., Annals of Nuclear Energy 69, 331 (2014)

[4] M.T. Pigni et al., Nuclear Data Sheets 123, 231 (2015)
[5] D. Rochman et al., Annals of Nuclear Energy 95, 125 (2016)

[6] N. Terranova et al., Nuclear Data Sheets 123, 225-230 (2015)

[7] N. Terranova et al., EPJ Web of Conferences 111, 09003 (2016)

[8] P. Archier et al., Nuclear Data Sheets 118, 488-490 (2014)

[9] U. Brosa et al., Physics Reports 4, 167 (1990)

[10] A.C. Wahl, "Systematics of Fission-Product Yields", LA-13928 (2002)

[11] D.G. Madland, T.R. England, Nuclear Science and Engineering 64, 859 (1977)

[12] B. Habert et al., Nuclear Science and Engineering 166, 276 (2010)

[13] P. Archier et al., PHYSOR-2014, Kyoto (2014)

[14] N. Terranova et al., PHYSOR-2016, Sun Valley, Idaho (2016)

[15] D. Schneider et al., PHYSOR-2016, Sun Valley, Idaho (2016) 